\title{
Effect of multiplicative noise on parametric instabilities
}

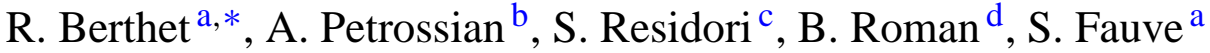 \\ a Laboratoire de Physique Statistique, Ecole Normale Supérieure, 24 rue Lhomond, 75231 Paris Cedex 05, France \\ b Yerevan State University, 1 A. Manoogian, 375025 Yerevan, Armenia \\ c Institut Non Linéaire de Nice, UMR 6618 CNRS-UNSA, 1361 Route des lucioles, 06560 Valbonne-Sophia Antipolis, France \\ ${ }^{\mathrm{d}}$ Faculté de St. Jérôme, IRPHE Service 252, 13397 Marseille Cedex 20, France
}

Received 19 October 2001; accepted 8 May 2002

\begin{abstract}
We report a study on the effect of external multiplicative noise on parametric instabilities using two different experimental systems: an electronic RLC circuit, parametrically pumped with a voltage-variable capacitor, and surface waves generated by vertically vibrating a layer of fluid (the Faraday instability). Both systems are forced by the superposition of a sinusoidal and a noisy component. We study the statistical properties of the response of both systems to noisy parametric forcing and compare them with theoretical predictions. When the detuning from parametric resonance is such that the bifurcation in the absence of noise is supercritical, both systems behave in the same way under the influence of noise. We find that the effect of noise is twofold: on one hand, it triggers the instability before its deterministic onset under the form of oscillatory bursts; on the other hand, it inhibits the nonlinearly saturated oscillatory response above the deterministic onset. When the detuning is such that the bifurcation is subcritical, we find that the two systems behave differently. In the case of the electronic oscillator, noise mostly triggers random transitions between the two states of the bistable region that exists in the absence of noise, whereas in the surface wave experiment new states are created by noise and the bistable region is strongly enlarged.

() 2002 Elsevier Science B.V. All rights reserved.
\end{abstract}

PACS: 05.40.-e; 47.20.-k; 47.20.Ky

Keywords: Bifurcations; Multiplicative noise; Parametric instabilities

\section{Introduction}

It has been known for a long time that random fluctuations of the control parameters of a dynamical system, or multiplicative noise, may generate surprising effects, such as stabilization by noise [1,2], different types of noise induced transitions [3,4], stochastic resonance (see for example [5]), etc. Although these effects have been predicted for a great variety of systems, only a few quantitative experiments have been

\footnotetext{
* Corresponding author.
}

performed on the effect of noise on the threshold of instabilities. Previous studies involve electronic oscillators [6], spin waves in ferrites and antiferromagnets $[7,8]$ and electroconvection in nematic liquid crystals $[9,10]$, but only the effect of noise on supercritical bifurcations has been considered so far. Only recently, experiments on the effect of noise on parametrically driven surface waves have been performed in the case of a subcritical bifurcation. New phenomena, such as noise-induced-bistability, have been reported [11].

Contrary to the case of additive noise, the basic solution of the deterministic system may be preserved 
in the case of multiplicative noise, and one can thus consider the effect of noise on its stability properties. The transition point can be shifted depending on the intensity of multiplicative noise. Stabilization, i.e. inhibition of the instability, has been reported in various situations $[1,2,6]$. Moreover, it has been found that multiplicative noise can modify the bifurcation diagram, generating new solutions that do not exist in the corresponding deterministic system. Examples of this kind of noise induced phase transitions can be found in $[3,12]$.

There are basic difficulties concerning the modelisation of the above phenomena, which are already apparent with the simplest possible example, a noisy pitchfork bifurcation,

$\dot{x}=[\mu+\xi(t)] x-x^{3}$,

where $\mu$ is the deterministic control parameter and $\xi(t)$ represents a Gaussian white noise with zero mean. First, the linear stability analysis of the $x=0$ solution is misleading, as it was shown in [13]: the linear growth rates of the moments become positive for $\mu_{n}<0, \mu_{n}$ depending on $n$, whereas it can be shown that when the nonlinear term is taken into account all the moments $\left\langle x^{n}\right\rangle$ go to zero for $\mu<0$, so that the bifurcation occurs for $\mu=0$ independently of $n$ $[1,14] .{ }^{1}$ On the contrary, if the most probable value of $x$ is taken as an order parameter, the bifurcation from zero occurs for $\mu>0$ and increasing with the noise intensity. ${ }^{2}$ As a consequence, the knowledge of the probability density function(PDF) of $x$ as a function of $\mu$ is required in order to fully describe the system.

Another problem is the interaction of noise with the damped modes of the deterministic system. Without noise, these modes are adiabatically eliminated in order to get the equation for the amplitude of the neutral mode, i.e. the order parameter. In the presence of noise, this elimination is no longer straightforward since the damped modes are continuously excited by noise. This

\footnotetext{
1 This inadequacy of the linear stability analysis in the presence of multiplicative noise has been also shown for more complex model equations.

2 Thresholds corresponding to different stability criteria have been compared in [15].
}

may influence even the linear stability problem as observed on the example of the Duffing equation

$\frac{1}{\gamma} \ddot{x}+\dot{x}=[\mu+\xi(t)] x-x^{3}$.

Without noise, this equation can be reduced to Eq. (1) in the vicinity of the bifurcation point $\mu=0$. This reduction is no longer possible in the presence of noise, and the bifurcation from zero is shifted to larger values of $\mu$ if $\left\langle x^{2}\right\rangle$ is taken as an order parameter in Eq. (2) contrary to the case of $(1)[1,2]$.

We study in this paper the effect of multiplicative noise on a parametric instability. Previous analytical and numerical studies have been performed on the Mathieu equation with a damping term

$\ddot{x}+2 \lambda \dot{x}+\omega_{0}^{2}[1+f(t)] x=0$,

where the forcing $f(t)$ involves both harmonic and noisy components

$f(t)=h \cos \Omega t+\xi(t)$.

In the case of forcing with noise only $(h=0),\langle x\rangle$ vanishes for $t \rightarrow \infty$ but the second moments of $x$ and $\dot{x}$ display a linear instability when the noise exceeds a critical value [16-19].

When harmonic and random excitations are simultaneously present, the problem of the inhibition or amplification of the parametric instability onset by noise has been addressed theoretically and numerically $[16,20]$ and an experimental study has been performed using an electronic parametric oscillator [6]. Although the experimental study displayed an inhibition of the parametric instability by noise, the linear stability analysis performed by Stratonovich showed that the threshold for the growth of the amplitude of the oscillations may be shifted in both directions, depending on the relative magnitude of the harmonic excitation amplitude, $h$, the power spectral density of the noise at $\Omega \simeq 2 \omega_{0}$, and the detuning from parametric resonance, $v=\Omega / 2-\omega_{0}$.

When a nonlinear term saturating the growth of the oscillation amplitude is added to the Mathieu equation, the PDF of the oscillation amplitude may be obtained [6]. It has been found that the inhibition of the parametric instability by noise could be explained only if 
the most probable value of the PDF of the oscillation amplitude is taken as an order parameter. However, this is not the quantity that was measured in the experimental study [6].

We report in this paper the measurement of the PDF of the oscillation amplitude with two different experimental systems undergoing a parametric instability with multiplicative noise. The first one is a vertically vibrated layer of fluid on which surface waves are generated by the Faraday instability $[21] .^{3}$ The second one is an electronic parametric oscillator, closely described by a Mathieu equation with a cubic nonlinearity [22]. The study of these two systems has been motivated by the fact that many damped modes are present in the one involving surface waves whereas this is not the case for the electronic oscillator. Thus, as said above, it is not obvious that both systems display the same response to noise.

This paper is organized as follows. In Section 2, we describe the two experiments. In Section 3, we recall the general features of parametric instabilities in the absence of noise. Section 4 is devoted to the study of the effect of noise on parametric instabilities in the supercritical regime. The subcritical case is considered in Section 5. The results are discussed and compared to earlier studies in Section 6.

\section{Description of the experimental systems}

\subsection{The electronic circuit}

We have first designed an electronic parametric oscillator in order to study analogically the response of a system governed by a nonlinear Mathieu equation to a forcing which involves both harmonic and noisy components. The electronic circuit is displayed in Fig. 1. Its consists of an RLC-oscillator where the inductance is realized using a gyrator filter [23], which gives a large and frequency independent inductance value ( $L \simeq 1 H$ ), and where $C$ is a voltage-variable capacitor (varactor diodes BB909A) [23]. The linear reso-

\footnotetext{
${ }^{3}$ Most of Faraday's experiments were repeated by Lord Rayleigh [21]. For more recent studies on the Faraday instability, see for instance, papers of Binks and Van de Water, and Kudrolli et al. [21].
}

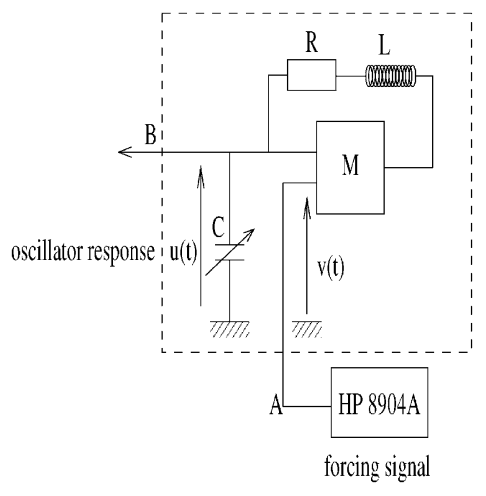

Fig. 1. Diagram of the electronic circuit.

nance frequency of the RLC circuit is $f_{0} \simeq 7.2 \mathrm{kHz}$, thus the capacitance of the varactor at low amplitude is $C_{0} \simeq 488 \mathrm{pF}$. The dependence of the capacity $C$ of varactor diodes on their charge $q$ is the dominant nonlinearity of the circuit. The use of pairs oppositely polarized varicaps keeps the symmetry $q \rightarrow-q$ of the Mathieu equation. Thus, we have to leading order $C \simeq C_{0}\left(1+\gamma q^{2}\right)$ with $\gamma>0$ [24].

The parametric forcing $v(t)$ is provided by a two-channel synthesizer HP8904A through an AD633 multiplier $M$. The charge $q$ of the capacity $C$ is thus governed by the equation

$\frac{\mathrm{d}^{2} q}{\mathrm{~d} t^{2}}+\frac{R}{L} \frac{\mathrm{d} q}{\mathrm{~d} t}+\frac{q}{L C}[1+k v(t)]=0$,

where $k$ is the gain of the analog multiplier $(k=$ $1 / 10 \mathrm{~V}$ in our case). We thus get

$\ddot{q}+2 \lambda \dot{q}+\omega_{0}^{2}[1+f(t)]\left(q-\gamma q^{3}\right)=0$,

with $2 \lambda=R / L, \omega_{0}^{2}=1 / L C_{0}$ and $f(t)=k v(t)$. We have $R \simeq 555 \Omega$ and the quality factor of the RLC circuit is $Q=L \omega_{0} / R \simeq 77$. The inductance value is calculated from the values of the gyrator's components. We can then deduce the capacitance value $C_{0}$ from the RLC resonance frequency. The global circuit resistance can be evaluated from the parametric threshold for a forcing frequency $\Omega / 2 \pi$. Thus, we can evaluate the quality factor of the RLC circuit, $Q=L \omega_{0} / R \simeq$ 77 , in good agreement with a direct measurement.

To avoid electromagnetic perturbations, the electronic circuit is enclosed in a metallic container 
playing the role of a Faraday cage. In addition, it is thermally regulated at $18 \pm 0.1{ }^{\circ} \mathrm{C}$ by circulating water. Thus, the measurements are reproducible within a $5 \%$ accuracy. For all results, the measurements errors are less than $1 \%$ for the parametric forcing parameters (frequency and amplitude) and within $5 \%$ for the response of the circuit.

\subsection{The surface waves experiment}

It is well known since Faraday [21] that standing surface waves can be generated by vertically vibrating a horizontal fluid layer. In the recent years, this pattern forming system has been widely studied (see for instance [25]). The linear stability analysis has been performed by Benjamin and Ursell [26] in the small viscosity limit. The deformation of the fluid surface has been described by normal modes obeying a Mathieu equation. Thus, in this limit the system is analogous to coupled parametric oscillators.

In the experiment described herein we study the effect of external multiplicative noise close to the bifurcation point of the instability. The schematical diagram of the experimental setup is shown in Fig. 2. Orientational degeneracy is avoided by using an elongated rectangular geometry so that the selected unstable mode is strongly confined along one direction and the excited wave can be considered as one-dimensional. The fluid container is an aluminum vessel of dimensions $15 \mathrm{~cm} \times 2 \mathrm{~cm} \times 1 \mathrm{~cm}$. It is rigidly attached to an electromagnetic vibration exciter (Brüjel

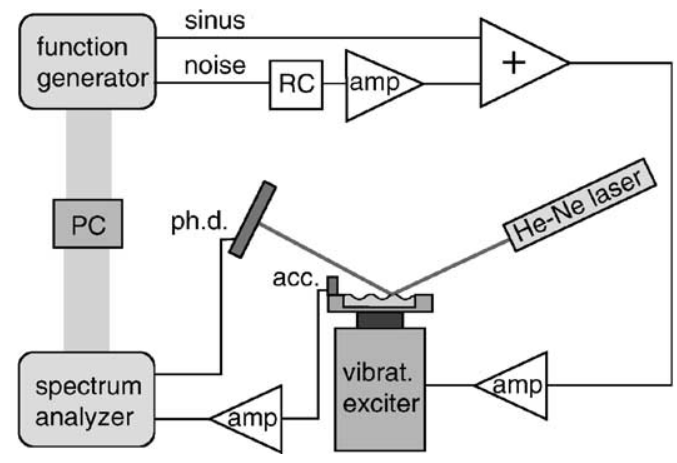

Fig. 2. Schematical diagram of the experimental setup (ph.d.: photodetector, acc.: accelerometer). and Kjaer 4809) which produces a clean vertical acceleration (horizontal acceleration less than $1 \%$ of the vertical one).

The vibration exciter is driven by a frequency synthesizer and the vertical acceleration is measured by a piezoeletric accelerometer and a calibrated charge amplifier. The fluid is distilled water. The fluid meniscus is eliminated by pinning the surface at the edges of the container and adjusting the fluid volume until the surface becomes flat everywhere [27]. To prevent liquid evaporation and contamination of the surface, the fluid container is closed with a Plexiglass plate and it is temperature controlled by circulating water and a thermal bath at $12.5 \pm 0.1^{\circ} \mathrm{C}$. In the absence of noise, thresholds are evaluated within a precision of a few percents. The stability of the experiment is checked to be quite good over times of the order of 1 day, which is the typical duration of each set of measurements in the presence of noise.

All the experiments are performed close to the instability threshold and the spatial pattern is a single mode without defects. Thus, the amplitude of the surface oscillations can be considered uniform along the whole cell length and can be measured by using a local optical detection [28]. The fluid is doped with black ink in order to avoid reflection from the bottom of the cell and an $\mathrm{He}-\mathrm{Ne}$ laser beam is focussed on the fluid surface. When the surface is flat, it reflects the light beam in one point. When the instability develops, the local slope of the surface is modulated by the wave, thus modulating the position of the reflected spot. In one period of oscillation, the reflected spot oscillates between two vertical extrema whose distance is proportional to the amplitude of the surface wave. A lens collects the reflected beam and focus it onto a photodetector that is essentially a rod of photoconductive material. The photodetector provides a voltage which is proportional to the position of the incoming beam, thus to the height of the surface. This way, the voltage signal follows the sinusoidal oscillation of the surface and the amplitude of the instability can be directly obtained by demodulating this signal.

A two-channel synthesizer HP8904A provides the sinusoidal and the noise signal. The noise is filtered, amplified and then summed to the sinusoidal 
excitation. Using a spectrum analyzer we check that the filtered noise is large band with a cutoff frequency around $1 \mathrm{kHz}$, so that it can be considered as a white noise around the typical frequencies of the sinusoidal signal $(\sim 60 \mathrm{~Hz})$. The two channel spectrum analyzer records the signals of the photodetector and of the accelerometer, so that the amplitude of the instability and the corresponding acceleration are monitored at the same time. When noise is added to the sinusoidal forcing, the acceleration in the reference frame of the fluid container is $g_{\text {eff }}=g+a \cos (\Omega t)+\eta(t)$, where $g$ is the acceleration of gravity. Thus, both the sinusoidal forcing and the noise $\eta(t)$, parametrically force gravity-capillary waves. The amplitude of each mode obeys a Mathieu equation in the linear approximation and in the limit of zero viscosity [26].

\subsection{Determination of the noise power spectral density and measurement of the oscillation amplitude}

The effect of multiplicative noise on a subharmonic parametric instability is characterized by the power spectral density of noise at the frequency $\Omega \simeq 2 \omega_{0}$ [16],

$\kappa(\Omega)=2 \int_{0}^{\infty}\left\langle\xi \xi_{\tau}\right\rangle \cos (\Omega \tau) \mathrm{d} \tau$.

For the electronic circuit, we measure the power spectral density by averaging the spectral power of noise over a frequency band centered in $\Omega$ and in the absence of the sinusoidal forcing. Since the noise spectrum is flat around $\Omega$, we suppose that this procedure gives a good estimation of the power spectral density $\kappa(\Omega)$. The amplitude $h$ of the sinusoidal component is set on the synthesizer. $\kappa(\Omega)$ is measured in $\mathrm{mV}^{2} / \mathrm{Hz}$. For both $h$ and $\kappa(\Omega)$, the normalization constant of the multiplier $k=0.1 \mathrm{~V}^{-1}$ should be taken into account.

For the surface wave experiment, we have to measure directly the acceleration to which is submitted the fluid container since we do not know precisely the response of the vibration exciter. This acceleration is the sum of the deterministic and the stochastic forcings. To separate the noise component, we have averaged the spectral power of the acceleration signal over a frequency band of $30 \mathrm{~Hz}$ on the left of the sinusoidal peak. Since the spectrum is flat around the peak, we suppose that this procedure gives a good estimation of the power spectral density $\kappa_{\mathrm{F}}(\Omega) . \kappa_{\mathrm{F}}(\Omega)$ is measured in $\mathrm{mV}^{2} / \mathrm{Hz}$, where $1 \mathrm{mV}$ corresponds to one unit of acceleration, i.e. to $1 \mathrm{~m} / \mathrm{s}^{2}$. The spectral power of the acceleration signal at $\Omega$ gives the rms value of the sinusoidal component $a_{\text {rms }}$. Note that because of the presence of the noisy component, the measured value of $a_{\mathrm{rms}}$ is Gaussian distributed.

The response $u(t)=q(t) / C$ of the electronic oscillator and the surface wave amplitude measured by the photodetector both display oscillations at frequency $\Omega / 2 \simeq \omega_{0}$ with an envelope varying randomly under the effect of noise. We have measured it with two different methods. In the surface waves experiment, we have performed the FFT of the photodetector signal on a frequency span of $400 \mathrm{~Hz}$ with a frequency resolution of $1 \mathrm{~Hz}$, thus over a time interval of $1 \mathrm{~s}$. This time scale corresponds to about 30 periods of the wave amplitude but is smaller than the characteristic duration of the wave bursts observed in the vicinity of the bifurcation in the presence of noise. Thus, the time dependence of the amplitude of the waves is well enough resolved in order to compute its PDF and its mean value. The spectral power of the photodetector signal at $\Omega / 2$ gives the modulus $|A|$ of the wave amplitude. With the electronic circuit, we have used a lock-in amplifier in order to record both the amplitude $|A|$ and the phase $\Phi$ of the oscillations. We have checked that both methods give the same result for the PDF of $|A|$.

\section{Parametric instabilities}

\subsection{Amplitude equation}

We recall here the basic features of parametric instabilities in the absence of noise. A canonical example is the parametric pendulum

$\ddot{x}+2 \lambda \dot{x}+\omega_{0}^{2}[1+f(t)] \sin x=0$,

where $f(t)=h \cos \Omega t$ is the parametric forcing, $2 \lambda$ is the damping and $\omega_{0}$ is the natural frequency. The linear stability of the solution $x=0$ is thus governed 
by the Mathieu equation (3) that displays parametric resonance whenever $n \Omega=2 \omega_{0}$, with $n$ integer. The strongest resonance occurs for $n=1$, i.e. $\Omega=2 \omega_{0}$. By the method of multiple scales, an amplitude equation can be obtained for the long time behavior of the unstable mode. In the limit of small dissipation, the temporal evolution of its slowly varying complex amplitude $\Psi$ obeys a normal form equation of the type [25]

$\partial_{t} \Psi=-(\lambda+\mathrm{i} v) \Psi+\mu \bar{\Psi}+\mathrm{i} \beta|\Psi|^{2} \Psi$,

where $v=\Omega / 2-\omega_{0}$ is the detuning from parametric resonance, $\mu=\omega_{0} h / 4$ is proportional to the amplitude of the forcing, and $\beta=-\omega_{0} / 4$ describes the leading order frequency correction with increasing oscillation amplitude. We write $\Psi=A \mathrm{e}^{\mathrm{i} \Phi}$, and get the stationary solutions of the amplitude equation

$\beta A^{2}=-v \pm \sqrt{\mu^{2}-\lambda^{2}}$.

For $v>0$ we have a supercritical bifurcation for $\mu_{\mathrm{c}}=\sqrt{\lambda^{2}+v^{2}}$. For $v<0$ the bifurcation is subcritical while the point $\left(v=0, \mu_{\mathrm{c}}=\lambda\right)$ is tricritical. The scaling behaviors above criticality display different features depending on $v$. If $\varepsilon=\mu-\mu_{\mathrm{c}}$ is the distance from threshold, the amplitude scales as $A \sim$ $\varepsilon^{1 / 4}$ for $v=0$ (bifurcation at the tricritical point); for the supercritical case $A \sim \varepsilon^{1 / 2}$. The subcritical case, as usual, has an unstable branch to which is associated an hysteresis cycle (see below).

\subsection{The electronic circuit as a parametric oscillator}

For the electronic circuit, the charge $q$ of the capacity $C$ is governed by Eq. (6) which is similar to the pendulum equation up to leading nonlinear order. Thus, the amplitude Eq. (9) governs the complex amplitude of oscillations of the voltage $u(t)=q / C$ measured in the vicinity of the parametric instability onset. The stability diagram of the electronic circuit parametrically forced in the vicinity of the strongest resonance $\Omega / 2 \pi \simeq 14.4 \mathrm{kHz}$, is displayed in Fig. 3 . The zero solution is linearly unstable above the resonance tongue (circles). As said above, the bifurcation

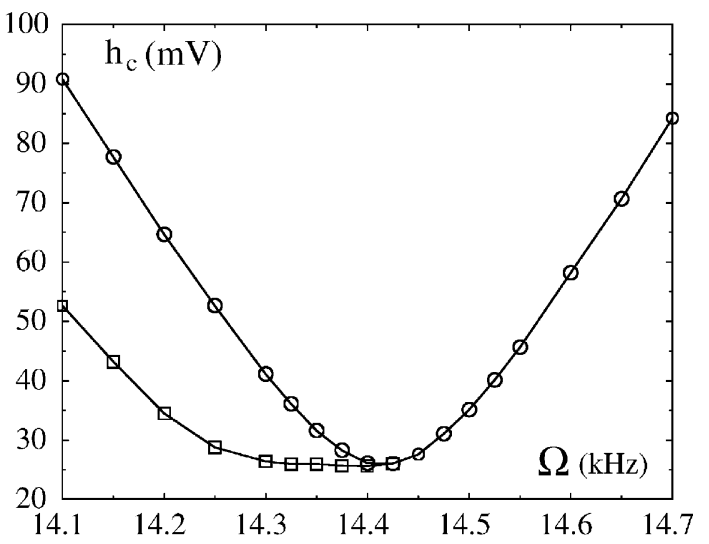

Fig. 3. Resonance tongue measured for the electronic circuit by varying the forcing frequency $\Omega$. The bifurcation is subcritical for negative detuning; the lower (respectively, higher) frontier of the bistability region correspond to the squares (respectively, circles).

from zero is supercritical for positive detunings and subcritical for negative ones. Thus, for negative detunings, finite amplitude oscillations exist below the linear threshold, until the forcing amplitude is decreased down to the nonlinear threshold (squares). These two curves delimitate the bistability region in which both the zero and oscillatory solutions are linearly stable.

\subsection{Parametric amplification of surface waves}

In the linear approximation and neglecting viscous dissipation, the fluid surface deformation can be decomposed in eigenmodes of amplitude $A_{k}$ and wavenumber $k$, each of them obeying a Mathieu-type equation [26]

$\ddot{A}_{k}+\omega_{0 k}^{2}\left(1+\frac{g_{1}(t)}{g+\sigma k^{2} / \rho}\right) A_{k}=0$,

where the effective acceleration in the reference frame of the fluid container is $g_{\text {eff }} \equiv g+g_{1}(t)$, with $g_{1}(t)=$ $a \cos (\Omega t)+\eta(t), g$ being the acceleration of gravity. $\sigma$ is the fluid surface tension and $\rho$ is its density.

The eigenfrequencies $\omega_{0 k}$ are related to the wavenumbers $k$ through the dispersion relation which, in the deep water limit, i.e. sufficiently large height $h$ of the fluid layer $(h \gg 2 \pi / k)$, reads

$\omega_{0}^{2}(k)=g k+\frac{\sigma}{\rho} k^{3}$. 
By comparing Eq. (11) to the standard form of the Mathieu equation (3), we have

$h \equiv \frac{a}{g\left[1+\left(k l_{\mathrm{c}}\right)^{2}\right]}$

and

$\xi(t) \equiv \frac{\eta(t)}{g\left[1+\left(k l_{\mathrm{c}}\right)^{2}\right]}$

where $l_{\mathrm{c}}=\sqrt{\sigma / \rho g}$ is the capillary length of the fluid.

Thus, in the Faraday experiment, the surface waves are parametrically driven. The most unstable mode is the one with a wavenumber such that $\omega_{0}(k)=\Omega / 2$. This resonance condition is in general only approximately satisfied because of boundary conditions such that a small frequency detuning $v$ is always present. Its sign cannot be easily predicted. We have characterized the response of the surface waves by exploring a frequency range of the sinusoidal forcing around $\Omega / 2 \pi=60 \mathrm{~Hz}$. The bifurcation displays supercritical features at $\Omega / 2 \pi=60 \mathrm{~Hz}$ whereas a subcritical behavior characterized by an hysteresis cycle is observed at $\Omega / 2 \pi=60.4 \mathrm{~Hz}$. In the following, when we refer to the supercritical or to the subcritical case we refer to these two values of $\Omega$, respectively.

When the fluid viscosity is taken into account in a phenomenological way, a Mathieu equation with a damping term of the form of Eq. (3) can be found for the modes $A_{k}$. However, only the viscous damping in the bulk of the flow can be described that way. The exact linear equation for $A_{k}$ involves also an integral term that traces back to viscous boundary layers which generate an additional damping of the waves [29]. However, viscous damping does not affect the qualitative features of the subharmonic parametric resonance except in particular situations [30].

Thus, as far as we can neglect the spatial variations of the wave amplitude, i.e. in the case of containers with a moderate aspect ratio, the surface deformation is described by a single standing wave mode oscillating at half the driving frequency. For fluids of small viscosity, the amplitude of this mode is governed by an equation analogous to the one derived for the parametric oscillator (9). The main difference between the two systems is that the Faraday experiment also in- volve many stable modes that may play a role in the presence of noise.

\section{Experimental results in the supercritical case}

\subsection{Noise induced amplification/inhibition of the oscillations}

We show in Fig. 4 typical time recordings of the oscillation amplitude of the parametrically amplified surface waves in the presence of noise. For small noise intensity, the stochastic part of the forcing adds small fluctuations around the deterministic value of the amplitude. These fluctuations are not symmetric around the deterministic value and they become more and more asymmetric for increasing noise amplitudes. For large values of noise, these fluctuations are so strong that they qualitatively change the behavior of the signal. The most probable value of the oscillation amplitude goes to zero so that it becomes difficult to recognize whether the sinusoidal forcing is above the deterministic threshold or not (compare Fig. $4 \mathrm{~b}$ and d). In this regime, the temporal behavior of the oscillation amplitude is characterized by bursts, corresponding to rare and intense events separated by "laminar" periods without oscillation. We have checked that when we observe a peak of the amplitude, the instability is homogeneously developed along the whole cell length. Therefore, the intermittent behavior is a purely temporal one and it is not related to the formation of spatial domains.

For the electronic circuit, the oscillation amplitude in the presence of noise behaves in a similar way. By means of a lock-in detection, we have also measured the phase of the temporal signals and we show the results in Fig. 5. We first observe that, above the deterministic threshold, the phase fluctuates around one of its two possible deterministic values, as long as the noise amplitude is not too large (Fig. 5a). Thus, the response of the oscillator is phase-locked when the oscillation amplitude remains large enough. When the noise intensity is increased, the amplitude almost vanishes between bursts and the phase jumps between its two possible deterministic values (Fig. 5b). Note 

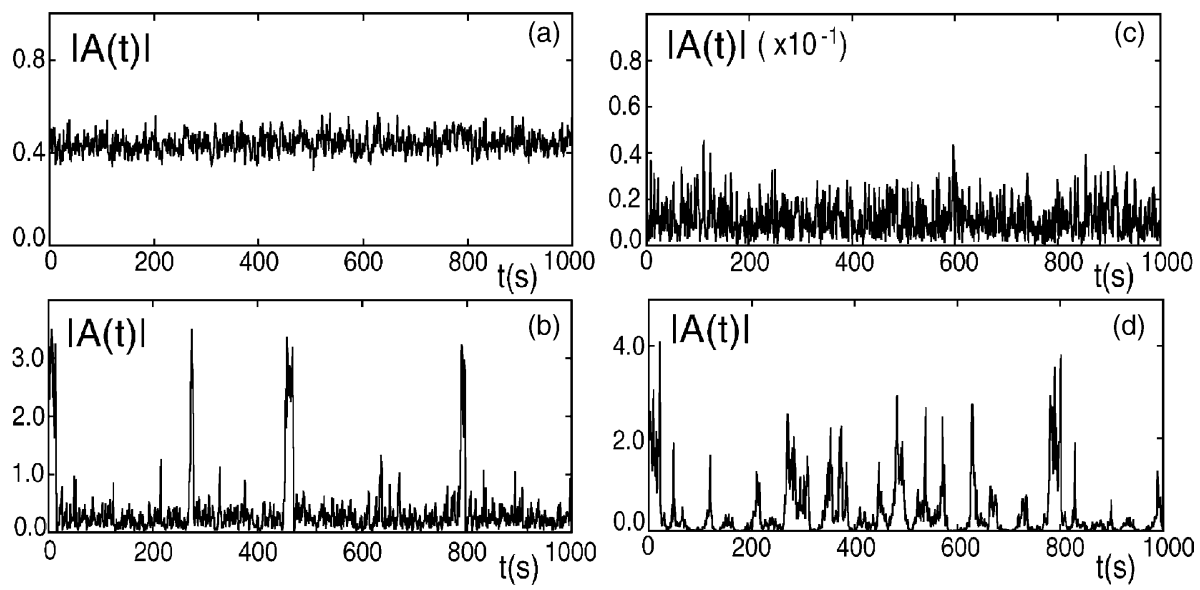

Fig. 4. Temporal evolution of the amplitude of oscillations in the presence of noise for the surface wave experiment. The amplitude is measured in arbitrary units. On the left, the average acceleration is $\left\langle a_{\mathrm{rms}}\right\rangle=5.4 \mathrm{~m} / \mathrm{s}^{2}$, corresponding to a forcing above the deterministic threshold. The noise intensity is $\kappa_{\mathrm{F}}(\Omega)=0.06 \mathrm{mV}^{2} / \mathrm{Hz}$ in (a) and $\kappa_{\mathrm{F}}(\Omega)=0.94 \mathrm{mV}^{2} / \mathrm{Hz}$ in (b). On the right, the average acceleration is $\left\langle a_{\mathrm{rms}}\right\rangle=5.0 \mathrm{~m} / \mathrm{s}^{2}$, corresponding to a forcing below the deterministic threshold. The noise intensity is $\kappa_{\mathrm{F}}(\Omega)=0.07 \mathrm{mV}^{2} / \mathrm{Hz}$ in $(\mathrm{c})$ and $\kappa_{\mathrm{F}}(\Omega)=1.19 \mathrm{mV}^{2} / \mathrm{Hz}$ in $(\mathrm{d})$.

however, that the phase remains locked during the bursts when the oscillation amplitude is large. The intermittent bursts in the oscillation amplitude are thus accompanied by intermittent phase jumps of $\pi$, which is the signature of a strong phase decorrelation of the signal. Phase jumps in the presence of noise are always observed below the deterministic threshold (Fig. 5c and d). However, here too the phase remains locked during large oscillatory bursts.

The average value of the oscillation amplitude as a function of the noise intensity is displayed in Fig. 6. A comparison with the bifurcation diagram in the absence of noise is not straightforward. Actually, the transition point seems to be shifted at a value smaller than the deterministic one, but just beyond the transition, noise reduces the amplitude of oscillations. Thus, below threshold, the average oscillation amplitude increases with noise whereas above threshold it decreases for increasing noise. The relative variation of the oscillation amplitude with respect to noise is of the same order of magnitude on both sides of the transition point (Fig. 6a and c). Therefore, the usual notion of threshold becomes ambiguous even in the presence of multiplicative noise. Considering only the supercritical regime, one may conclude that the parametric instability is inhibited by noise as said in reference [6]. On the other hand, considering the increase of the mean oscillation amplitude with increasing noise below the deterministic threshold, we may conclude that the instability onset is shifted towards lower values in the presence of noise. It is thus not possible in general to state whether noise amplifies or inhibits the oscillations. This ambiguity traces back to the choice of the order parameter of the transition in the presence of noise. One may indeed take, the mean value of the oscillation amplitude, the most probable value of the amplitude, the phase coherent part of the amplitude, as different possible choices, and get different answers. ${ }^{4}$ In order to resolve the ambiguity, it is necessary to look at the statistical properties of the signals and hence to measure the whole PDF of the amplitude of oscillations.

\subsection{Statistical properties of oscillations in the presence of noise}

The PDFs of the oscillation amplitude measured with the electronic circuit for different noise intensities and at a fixed sinusoidal forcing are displayed in Fig. 7. When the sinusoidal forcing is above the

\footnotetext{
${ }^{4}$ Other stability criteria can also been considered, see [15].
} 

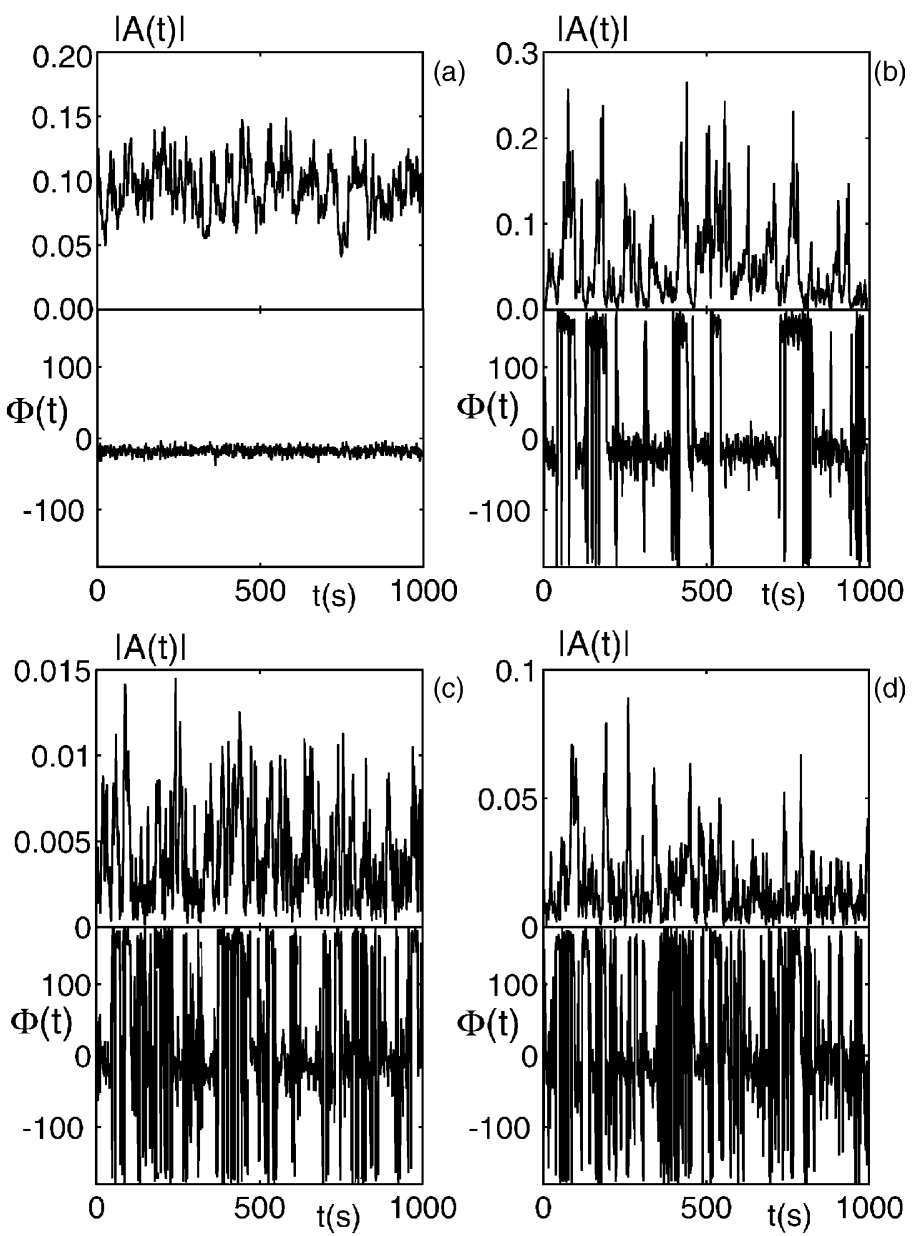

Fig. 5. Temporal evolution of the amplitude $|A|$ and the phase $\Phi$ of the oscillations measured with the electronic circuit: (a) and (b) $h=31.25 \mathrm{mV}$, i.e. above the deterministic threshold for a noise intensity: (a) $\kappa(\Omega)=0.94 \mathrm{mV}^{2} / \mathrm{Hz}$ and (b) $\kappa(\Omega)=8.25 \mathrm{mV} / \mathrm{Hz}$; (c) and (d) $h=24.5 \mathrm{mV}$, i.e. below the deterministic threshold for a noise intensity: (c) $\kappa(\Omega)=0.94 \mathrm{mV}^{2} / \mathrm{Hz}$ and $(\mathrm{d}) \kappa(\Omega)=8.25 \mathrm{mV}^{2} / \mathrm{Hz}$.

deterministic threshold, the PDFs behave according to the theoretical predictions [6,16]. For small noise, the amplitude fluctuates in the vicinity of its deterministic value and the PDF is almost Gaussian. When the noise intensity is increased, the PDF becomes asymmetric. For a critical noise intensity, there is a transition at which the most probable value vanishes (see Fig. 7a). We can compare our results with the analytical prediction obtained from the stationary solution of the Fokker-Planck equation with the assumption that the oscillator response is phase-locked [6]. As we showed above, this assumption is correct above the deterministic threshold for small enough noise intensity. The
PDF is then of the form

$P(X) \propto X^{(a-1)} \exp (-b X)$,

where $X=|A|^{2}, a$ and $b$ depends on the characteristics of the electrical circuit. We did not try to calculate $a$ and $b$ as functions of the circuit parameters, but it is clear from Eq. (15) that $\langle X\rangle=a / b$ and $\sigma(X)=$ $\sqrt{a} / b$. We have determined $\langle X\rangle$ and $\sigma$ from the experimental data and then we have inserted these values in the above expression of the PDF. By doing this and normalizing the PDF to one, we find that this prediction (full lines in Fig. 7a) is in agreement with our experimental data. 


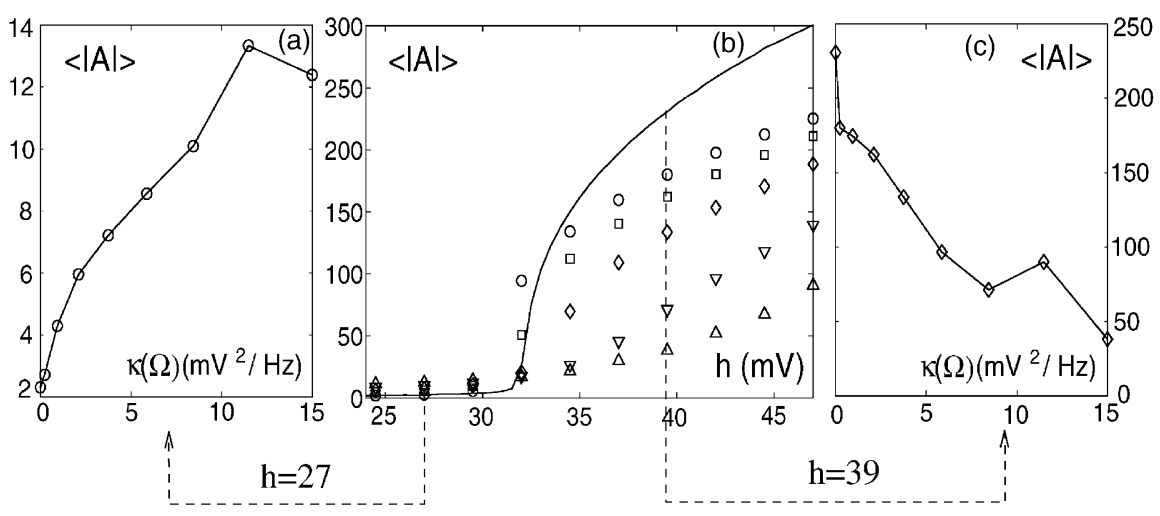

Fig. 6. Average value of the oscillation amplitude measured with the electronic circuit as a function of the noise intensity: (a) $h=27 \mathrm{mV}$ and (c) $h=39 \mathrm{mV}$ and as a function of $h$ for different noise intensities (b): $\kappa(\Omega)=0 \mathrm{mV}^{2} / \mathrm{Hz}$ (solid line), $\kappa(\Omega)=0.38 \mathrm{mV}^{2} / \mathrm{Hz}($ circles), $\kappa(\Omega)=2.10 \mathrm{mV}^{2} / \mathrm{Hz}$ (squares), $\kappa(\Omega)=3.75 \mathrm{mV}^{2} / \mathrm{Hz}$ (diamonds), $\kappa(\Omega)=8.25 \mathrm{mV}^{2} / \mathrm{Hz}$ (reversed triangles), $\kappa(\Omega)=15.00 \mathrm{mV} / \mathrm{Hz}$ (triangles).

When the sinusoidal forcing is below the deterministic threshold, the PDFs becomes wider and wider as we add more and more noise. Nevertheless, the most probable value remains zero as a consequence of the fact that the system spends most of the time close to the fixed point corresponding to the stable solution in the absence of noise. The large events being rare, the average value does not deviate considerably from zero.

\section{Experimental results in the subcritical case}

\subsection{Experimental results for the electronic circuit}

As said above, the parametric oscillator undergoes a subcritical bifurcation in the case of negative detuning. Time recordings of the oscillation amplitude and phase in the presence of noise are displayed in Fig. 8.
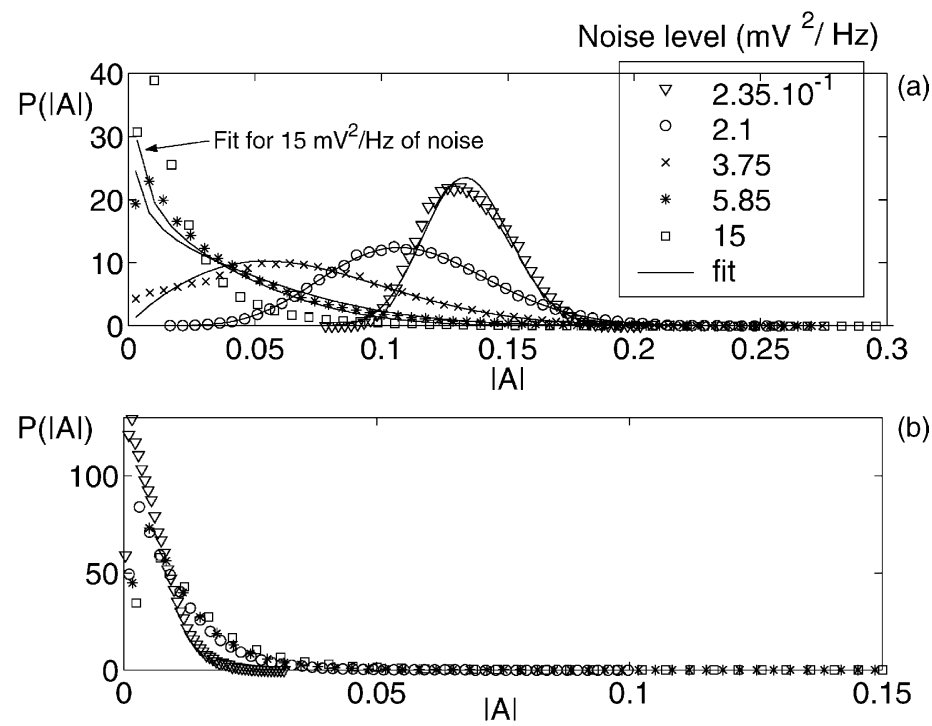

Fig. 7. PDFs of the amplitude of oscillations measured with the electronic circuit for increasing values of noise. (a) $h=34.5 \mathrm{mV}$, i.e. above the deterministic threshold. The full lines correspond to the fit with Eq. (15). (b) $h=29.5 \mathrm{mV}$, i.e. below the deterministic threshold. 

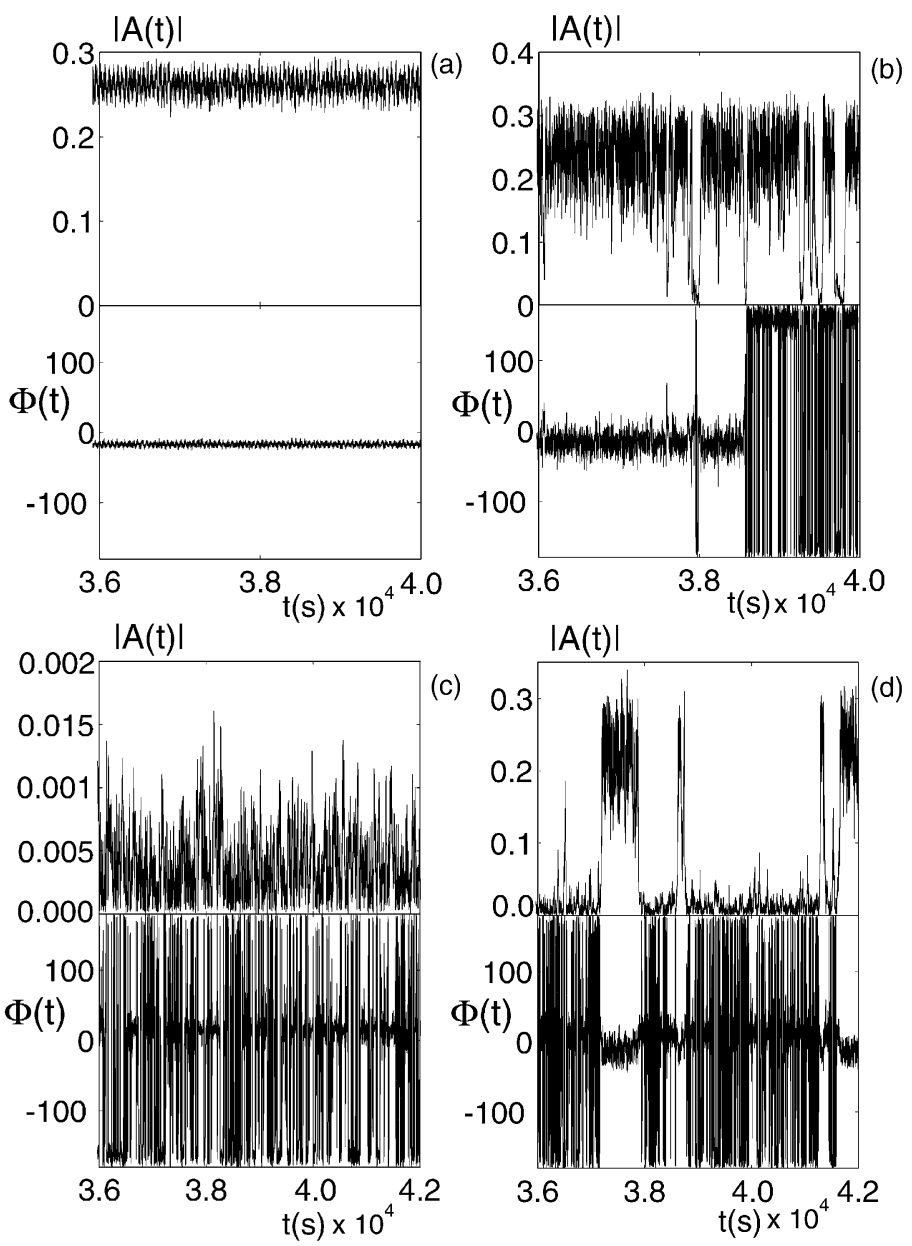

Fig. 8. Temporal evolution of the amplitude $|A|$ and the phase $\Phi$ of the oscillations measured with the electronic circuit in the case of a subcritical bifurcation: (a) and (b) $h=32 \mathrm{mV}$, i.e. above the deterministic linear threshold for a noise intensity: (a) $\kappa(\Omega)=0.24 \mathrm{mV} / \mathrm{Hz}^{2}$ and (b) $\kappa(\Omega)=5.85 \mathrm{mV}^{2} / \mathrm{Hz}$; (c) and (d) $h=29.5 \mathrm{mV}$, i.e. below the deterministic linear threshold for a noise intensity: (c) $\kappa(\Omega)=0.24 \mathrm{mV} / \mathrm{Hz}$ and $(\mathrm{d}) \kappa(\Omega)=3.75 \mathrm{mV}^{2} / \mathrm{Hz}$.

Above the linear threshold, at first sight, the behavior looks similar to the one observed in the supercritical case. For small noise intensities, the amplitude and the phase fluctuate in the vicinity of their deterministic values (Fig. 8a). For larger noise intensities, the mean amplitude decreases and phase jumps are observed when the instantaneous value of the amplitude goes to zero (Fig. 8b). However, the PDFs do not display the continuous shift to zero of the most probable value as in the supercritical case. Two local maxima are observed and their positions are almost not affected by the noise intensity (Fig. 9a). The larger one traces back to the bifurcated limit cycle of the deterministic system; its amplitude is decreased in the presence of noise as in the supercritical case. The smaller one corresponds to the zero solution of the deterministic system which is slightly increased in amplitude under the effect of noise, again as in the supercritical case. However, above the deterministic linear threshold, the zero solution is no longer stable in the absence of noise. The existence of the smaller maximum of the PDF, thus means that the zero solution is "stabilized" by noise.

The effect of noise on the time recordings of the oscillation amplitude and phase in the bistability region 
of the deterministic system, are displayed in Fig. 8c and $d$. For small enough noise, the amplitude fluctuates in the vicinity of zero and the phase undergoes erratic jumps as in the supercritical case (Fig. 8c). When the noise intensity increases, transitions to the finite mean amplitude limit cycle are generated, and the oscillator displays random jumps between the zero and non-zero states which are both linearly stable in the deterministic system. The phase is locked when the oscillation amplitude is finite (Fig. 8d). The corresponding PDFs display two local maxima for zero and finite amplitudes (Fig. 9b). When the noise intensity is increased, the probability of the finite amplitude state decreases, showing again that large noise tends to "stabilize" the zero solution. However, the position of the local maxima of the PDF are not affected by the intensity of noise.

In conclusion, the effect of noise on a parametrically amplified limit cycle depends on the super or subcritical nature of the bifurcation, even out of the bistability range of the deterministic system. Instead of the continuous shift to zero of the finite amplitude maximum of the PDF observed in the supercritical case, two local maxima of the PDF of the oscillation amplitude are observed in the subcritical case. Although, these two maxima trace back to the two metastable states of the deterministic system, their amplitude and their relative stability are affected by noise. As in the super- critical case, the bifurcation diagram in the presence of noise strongly depends on the choice of the order parameter. If the mean value of the oscillation amplitude is chosen, the bifurcation remains first-order for a low external noise level, but becomes continuous at high enough noise (Fig. 10 a). This may be analogous in the time domain, to the phenomenon observed in phase transitions, where it has been shown that microscopic random impurities or other type of spatial disorder, may produce rounding of a first-order phase transition [31-34]. If the most probable value of the PDF is taken as an order parameter, the transition obviously remains discontinuous but the bistability range is modified by noise (Fig. 10b).

\subsection{Noise-induced-bistability of parametric surface waves}

We study in this section the effect of noise on the parametric amplification of surface waves through a subcritical bifurcation and we emphasize the differences with respect to the electronic oscillator. Time recordings of the amplitude of the waves in the presence of noise are displayed in Fig. 11 as the sinusoidal forcing is increased. The frequency of the parametric excitation is $\Omega / 2 \pi=60.4 \mathrm{~Hz}$, corresponding to a negative detuning (roughly a tenth of $\mathrm{Hz}$ ). Consequently, the bifurcation without noise is subcritical
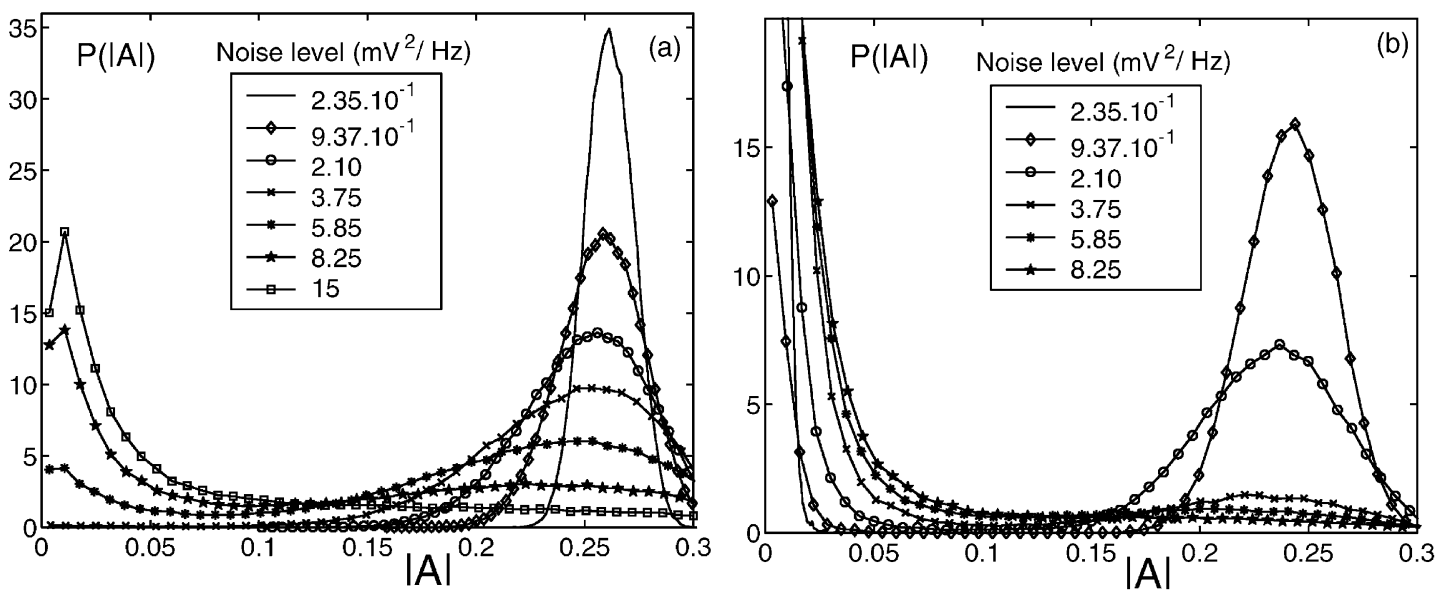

Fig. 9. PDFs of the oscillation amplitude as a function of the noise intensity, measured with the electronic circuit in the subcritical case: (a) $h=32 \mathrm{mV}$, i.e. above the deterministic linear threshold; (b) $h=29.5 \mathrm{mV}$, i.e. below the deterministic linear threshold. 

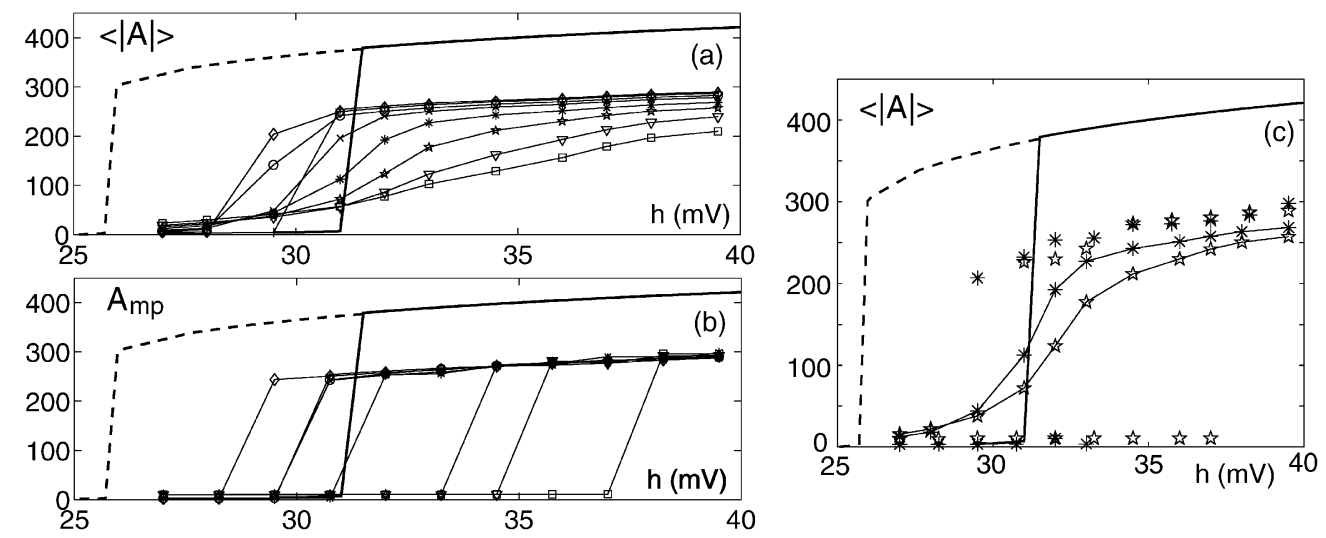

Fig. 10. Bifurcation diagram for: (a) the average value $\langle|A|\rangle$ and (b) the most probable value $A_{\mathrm{mp}}$ of the oscillation amplitude measured with the electronic circuit in the subcritical case and for different noise intensities. We report in (c) the two most probable values of the corresponding PDFs together with their average values. Solid (respectively, dashed) lines represent the bifurcation diagrams in the absence of noise for increasing (respectively, decreasing) forcing. $\kappa(\Omega)=0.94 \mathrm{mV}^{2} / \mathrm{Hz}$ (diamonds), $\kappa(\Omega)=2.10 \mathrm{mV}^{2} / \mathrm{Hz}($ circles), $\kappa(\Omega)=3.75 \mathrm{mV}^{2} / \mathrm{Hz}$ (crosses), $\kappa(\Omega)=5.85 \mathrm{mV}^{2} / \mathrm{Hz}$ (stars), $\kappa(\Omega)=3.75 \mathrm{mV}^{2} / \mathrm{Hz}$ (pentagons), $\kappa(\Omega)=11.48 \mathrm{mV}^{2} / \mathrm{Hz}($ triangles), $\kappa(\Omega)=15.00 \mathrm{mV}^{2} / \mathrm{Hz}$ (squares). Thin lines are just guides for the eyes.

and the wave amplitude abruptly jumps to a finite value for a rms acceleration $a_{\mathrm{c}}=5.8 \pm 0.1 \mathrm{~m} / \mathrm{s}^{2}$. The noise intensity is $\kappa_{\mathrm{F}}(\Omega)=0.10 \mathrm{mV}^{2} / \mathrm{Hz}$. We can see

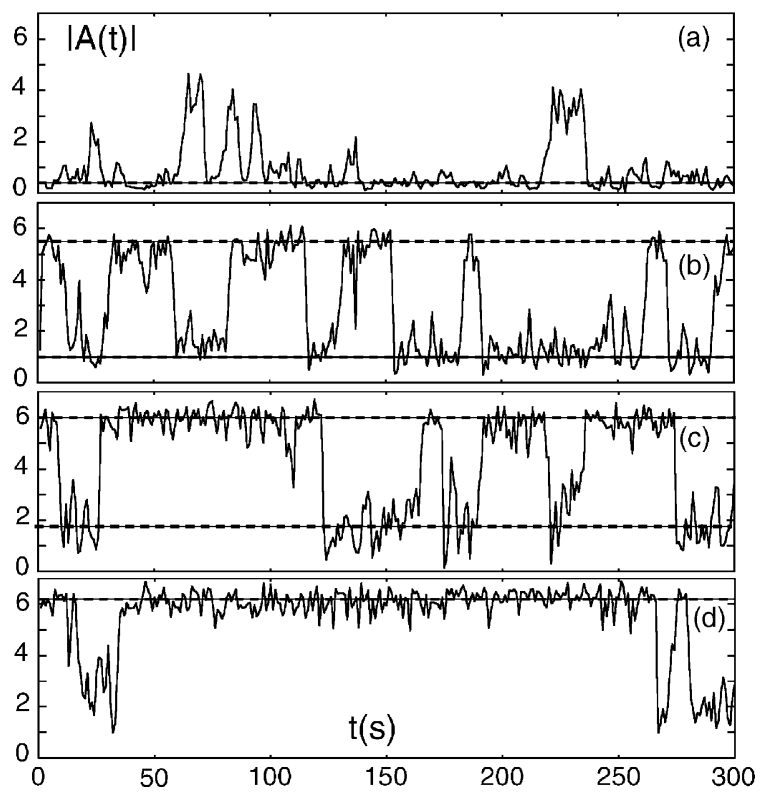

Fig. 11. Temporal evolution of the wave amplitude in the subcritical case for a noise intensity $\kappa_{\mathrm{F}}(\Omega)=0.10 \mathrm{mV}^{2} / \mathrm{Hz}$. The sinusoidal forcing corresponds to an average acceleration $\left\langle a_{\mathrm{rms}}\right\rangle=5.7,5.9,6.2,6.4 \mathrm{~m} / \mathrm{s}^{2}$ in (a), (b), (c) and (d), respectively.
(Fig. 11a) that noise triggers oscillatory bursts before the deterministic threshold, as in the supercritical case. The presence of this rare but large events makes the average value of the wave amplitude different from zero before the deterministic threshold. Note however, that the most probable value is zero. When the average acceleration is increased the amplitude begins to switch between two values indicated by the two dotted lines in Fig. 11b and c. Both values are increasing when the amplitude of the sinusoidal forcing is increased, and the system spends more and more time in the vicinity of the largest one. The PDFs corresponding to the time recordings of Fig. 11 are displayed in Fig. 12. At first sight, one may think that, as in the case of the electronic oscillator, the effect of noise consists of triggering transitions between the two metastable states that exist in the vicinity of a subcritical bifurcation. We emphasize below that this is not the case.

The amplitude of the waves measured in the absence of noise is shown with circles in the bifurcation diagram displayed in Fig. 13. As said above, there is an abrupt jump from zero to finite amplitude for $a_{\mathrm{c}}=$ $5.8 \pm 0.1 \mathrm{~m} / \mathrm{s}^{2}$. The subcritical nature of the bifurcation is due to negative detuning. The bistable region in the vicinity of this subcritical bifurcation is rather 
(a)

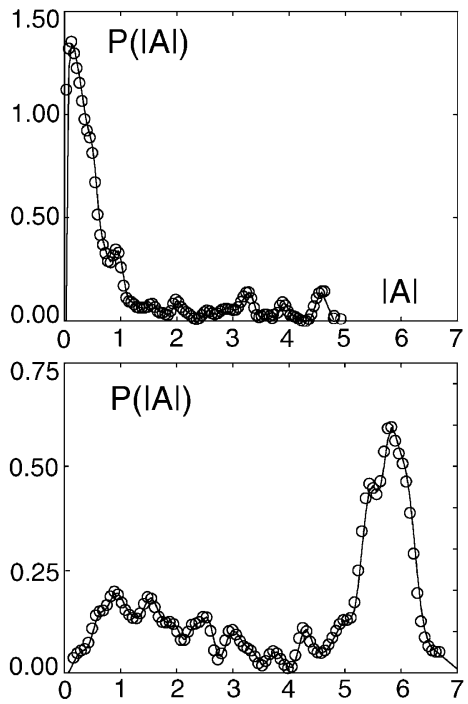

(b)

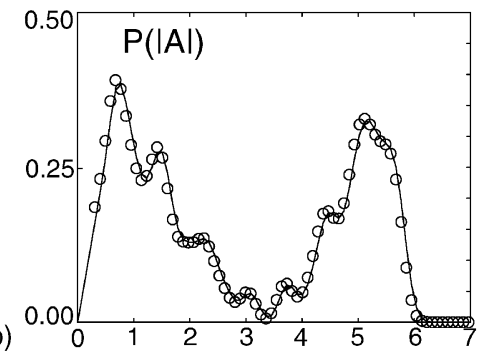

(d)

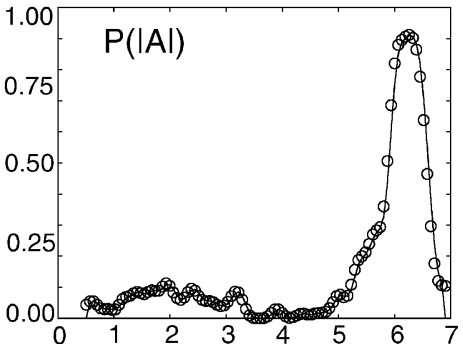

Fig. 12. PDFs of the wave amplitude in the subcritical case for a noise intensity $\kappa_{\mathrm{F}}(\Omega)=0.10 \mathrm{mV}^{2} / \mathrm{Hz}$ and for different values of the deterministic forcing: $\left\langle a_{\mathrm{rms}}\right\rangle=5.7,5.9,6.2,6.4 \mathrm{~m} / \mathrm{s}^{2}$ in (a), (b), (c) and (d), respectively.

small but is strongly enlarged in the presence of noise. The most probable values of the wave amplitude in the presence of noise $\left(\kappa_{\mathrm{F}}(\Omega)=0.10 \mathrm{mV}^{2} / \mathrm{Hz}\right.$ as in Fig. 11) are plotted in Fig. 13. We observe a lower branch that continuously increases from zero and an

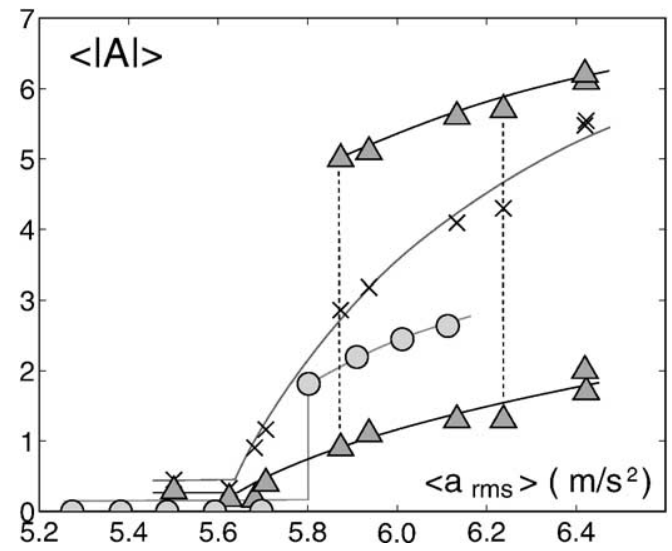

Fig. 13. Bifurcation diagram for the amplitude of the surface waves in the subcritical case. The wave amplitude $|A|$ is measured in arbitrary units. Circles are measured in the absence of noise. Crosses correspond to the average value $\langle|A|\rangle$ measured for a noise intensity $\kappa_{\mathrm{F}}(\Omega)=0.10 \mathrm{mV}^{2} / \mathrm{Hz}$. For the same set of measurements triangles represent the most probable values of the corresponding PDF. Lines are guides for the eyes. upper branch which exists for a rms acceleration above $a_{1}=5.9 \pm 0.1 \mathrm{~m} / \mathrm{s}^{2}$. Thus, two branches of solutions, displaying a bistable region, are created by noise. The two dotted lines mark the beginning of the bistable region and the point $a_{2}=6.2 \pm 0.1 \mathrm{~m} / \mathrm{s}^{2}$ above which the probability of visiting the lower branch has decreased to ten percent of the probability of visiting the upper one.

The average wave amplitude $\langle|A|\rangle$ is shown with crosses in Fig. 13. We first observe that $\langle|A|\rangle$ increases continuously from zero when the average acceleration, $\left\langle a_{\mathrm{rms}}\right\rangle$, is increased. However, $\langle|A|\rangle$ does not interpolate between the two branches of the deterministic bifurcation diagram as it would do if the only effect of noise were to generate random transitions between these two states. It of course interpolates between the two branches for the most probable value of the wave amplitude. The same bifurcation diagram is displayed in Fig. 14 for a larger noise intensity $\left(\kappa_{\mathrm{F}}(\Omega)=0.18 \mathrm{mV}^{2} / \mathrm{Hz}\right.$.) The behavior is qualitatively the same but, rather surprisingly, the noise induced bistable region is shifted to higher values of the average acceleration. The value of the slope of $\langle|A|\rangle$ versus the average acceleration decreases. 


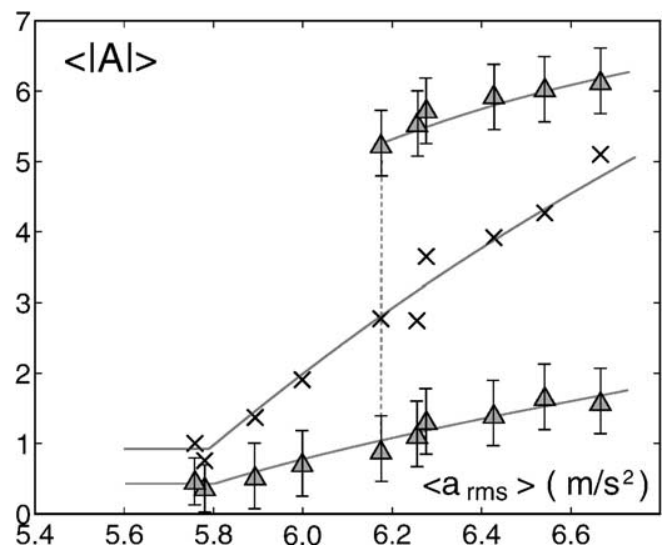

Fig. 14. Bifurcation diagram (crosses) of the average wave amplitude in the subcritical case for $\kappa_{\mathrm{F}}(\Omega)=0.18 \mathrm{mV}^{2} / \mathrm{Hz}$. Triangles represent the most probable values of the corresponding PDF. Lines are guides for the eyes.

\section{Conclusions}

The effect of multiplicative noise on parametric instabilities has been considered for a long time. Both an analytical linear stability analysis of the zero solution of the Mathieu equation with a forcing involving harmonic and noisy components [16], and an experiment with an electronic parametric oscillator [6], have been performed. Our experimental results differ in several important aspects from the previous studies: first, concerning the experimental measurements, we have measured both the amplitude and the phase of the oscillations in the presence of noise. Second, we have studied the PDF of the oscillation amplitude and compared it with the theoretical prediction made with the assumption of phase-locked oscillations. Second, we have also considered the effect of noise on a subcritical bifurcation and compared a low dimensional dynamical system (the electronic oscillator) with a spatially extended one which involves many modes that may be excited by noise.

The effect of noise on parametric amplification is twofold. When the system is below the deterministic threshold for the instability, noise triggers the onset of oscillations. On the other hand, once the oscillations are developed, noise decreases their amplitude. The first behavior may be described by the linear study of Stratonovich in the case of small noise [16]. Indeed, the threshold can be lowered by noise depending on the relative strength of noise, forcing and detuning from resonance. But, once an oscillatory regime is developed, the nonlinearity becomes important. In this case, the dominant effect is the phase decorrelation induced by noise. This is equivalent to introduce detunings bringing the oscillator out of resonance and thus, decreasing its amplitude.

We have shown that the effect of noise on a parametrically amplified limit cycle depends on the super or subcritical nature of the bifurcation. Instead of the continuous shift to zero of the finite amplitude maximum of the PDF observed in the supercritical case, two local maxima of the PDF of the oscillation amplitude are observed in the subcritical case. In the case of the electronic oscillator, i.e. a low dimensional dynamical system, these two maxima trace back to the two metastable states of the deterministic system. In the case of surface waves new states can be "stabilized" by noise and the bistable region of the deterministic system may be strongly enlarged by multiplicative noise. We note that "noise-enhanced multistability" has been reported in a simple model of coupled oscillators but also requires additive noise [35]. The structure of this model being of a very different nature than the one involved in parametric amplification, we expect that noise-induced bistability can be observed with other subcritical pattern-forming instabilities.

\section{Acknowledgements}

This work has benefited of the financial supports of INTAS contract 1672 and CNRS GDR G2280.

\section{References}

[1] A. Schenzle, H. Brand, Phys. Rev. A 20 (1979) 1628; R. Graham, A. Schenzle, Phys. Rev. A 26 (1982) 1676.

[2] M. Lücke, F. Schank, Phys. Rev. Lett. 54 (1985) 1465.

[3] W. Horsthemke, R. Lefever, Noise-induced Transitions, Springer, Berlin, 1984.

[4] C. Van den Broeck, J.M.R. Parrondo, R. Toral, Phys. Rev. Lett. 73 (1994) 3395.

[5] L. Gammaitoni, P. Hänggi, P. Jung, F. Marchesoni, Rev. Mod. Phys. 70 (1998) 223. 
[6] T. Kawakubo, S. Kabashima, Y. Tsuchiya, Progr. Theor. Phys. 64 (Suppl.) (1978) 150;

S. Kabashima, S. Kogure, T. Kawakubo, T. Okada, J. Appl. Phys. 50 (1979) 6296.

[7] V.V. Zautkin, B.I. Orel, V.B. Cherepanov, Sov. Phys. JETP 58 (1983) 414.

[8] V. Lvov, Wave Turbulence Under Parametric Excitation, Springer, Berlin, 1994.

[9] T. Kawakubo, A. Yanagita, S. Kabashima, J. Phys. Soc. Jpn. 50 (1981) 1451.

[10] T. John, R. Stannarius, U. Behn, Phys. Rev. Lett. 83 (1999) 749.

[11] S. Residori, R. Berthet, B. Roman, S. Fauve, Phys. Rev. Lett. 88 (1) (2002) 24502.

[12] C. Van den Broeck, J.M.R. Parrondo, J. Armero, A. Hernandez-Machado, Phys. Rev. E 49 (1994) 2639.

[13] M. San Miguel, R. Toral, in: E. Tirapegui et al. (Eds.), Instabilities and Nonequilibrium Structures, vol. VI, Kluwer, Dordrecht, 2000.

[14] A. Becker, L. Kramer, Phys. Rev. Lett. 73 (1994) 955.

[15] U. Behn, A. Lange, T. John, Phys. Rev. E 58 (1998) 2047.

[16] R.L. Stratonovich, Topics in the Theory of Random Noise, Gordon and Breach, London, 1963.

[17] R.C. Bourret, Physica 54 (1971) 623; R.C. Bourret, U. Frisch, A. Pouquet, Physica 65 (1973) 303.

[18] N.G. van Kampen, Stochastic Processes in Physics and Chemistry, North-Holland, Amsterdam, 1981.

[19] P.S. Landa, A.A. Zaikin, Phys. Rev. E 54 (1996) 3535.

[20] W. Zhang, J. Casademunt, J. Vinals, Phys. Fluids A 5 (1993) 3147.
[21] M. Faraday, Philos. Trans. Roy. Soc. London 121 (1831) 319; Lord Rayleigh, Philos. Mag. 16 (1883) 50;

D. Binks, W. van de Water, Phys. Rev. Lett. 78 (1997) 4043; A. Kudrolli, M.C. Abraham, J.P. Gollub, Phys. Rev. E 63 (2001) 026208.

[22] R. Berthet, A. Petrossian, B. Roman, Am. J. Phys. 70 (2002) 744;

R. Berthet, S. Residori, B. Roman, S. Fauve, L'instabilité paramétrique en présence de bruit multiplicatif, Rencontre du Non-linéaire, Paris Onze Editions, 2000, p. 265.

[23] P. Horowitz, W. Hill, The Art of Electronics, Cambridge University Press, Cambridge, 1989.

[24] L. Falk, Am. J. Phys. 47 (1979) 325.

[25] S. Fauve, Pattern forming instabilities, in: C. Godrèche, P. Manneville (Eds.), Hydrodynamics and Nonlinear Instabilities, Cambridge University Press, Cambridge, 1998.

[26] T.B. Benjamin, F. Ursell, Proc. Roy. Soc. London A 225 (1954) 505.

[27] T.B. Benjamin, C. Scott, J. Fluid. Mech. 92 (1979) 241.

[28] S. Douady, Instabilité paramétrique d'ondes de surface, Ph.D. Thesis, ENS de Lyon, 1989.; S. Douady, J. Fluid Mech. 221 (1990) 3383-3409.

[29] U. Nam Hong, Bull. Russian Acad. Sci., Phys. 57 (Suppl.) (1993) 131.

[30] K. Kumar, Proc. Roy. Soc. London A 452 (1996) 1113.

[31] Y. Imry, S.-K. Ma, Phys. Rev. Lett. 35 (1975) 1399.

[32] Y. Imry, M. Wortis, Phys. Rev. B 19 (1979) 3580.

[33] M. Aizenman, J. Wehr, Phys. Rev. Lett. 62 (1989) 2503.

[34] J. Cardy, J.L. Jacobsen, Phys. Rev. Lett. 79 (1997) 4063.

[35] S. Kim, S.H. Park, C. Su Ryu, Phys. Rev. Lett. 78 (1997) 1616. 